\title{
Business of Local Fruit and Vegetables in Jember District as a Support of Food Security, Indonesia
}

\author{
Sawitri Komarayanti $^{1}$, Wiwik Suharso ${ }^{2}$, Elfien Herrianto ${ }^{3}$ \\ ${ }^{1.3}$ Biology Education Studies Program, Faculty of Teacher Training and Education Universitas Muhammadiyah \\ Jember, Indonesia \\ ${ }^{2}$ Informatics Engineering Program, Faculty of Engineering Universitas Muhammadiyah Jember, Indonesia \\ email: sawitrikomarayanti@unmuhjember.ac.id
}

\begin{abstract}
Local fruits and vegetables contribute to food security in the area. Efforts to gather information about local fruits and vegetables is very important. This is due to the diversity of local fruit and vegetables are endangered due to the penetration of imported fruits and vegetables that increasingly dominate, changing consumption patterns and land use. This study examines the diversity of local fruits and vegetables in Jember. The research method using exploratory survey which aims to gather information with a qualitative approach. The research instrument is observation sheet, interview and documentation. The survey area covers 31 districts in Jember, namely:Subdistrict Kencong, Gumukmas, Puger, Wuluhan, Ambulu, Tempurejo, Silo, Mayang, Mumbulsari, Jenggawah, Ajung, Rambipuji, Balung, Umbulsari, Semboro, Jombang, Sumberbaru, Embankment, Bangsalsari, Panti, Sukorambi, Arjasa, Pakusari, Kalisat, Ledokombo, Sumberjambe, Sukowono, Jelbuk, Kaliwates, Sumbersari and Patrang, Respondents were interviewed are traders of fruit and vegetables in the market and the centers of the fruit. The results of this exploratory study found that 46 kinds of local fruits and 52 kinds of local vegetables. $52.2 \%$ met the local fruit is cultivated, ie 24 types of local fruits such as:Avocado, Grapes, Belimbing, Duku, Durian, Guava, Guava Water, Orange Semboro, Orange Large, longan, mango, mangosteen, Melinjo, jackfruit, pineapple, papaya, banana, rambutan, Salak, Sawo, passionfruit, soursop and breadfruit. $30.8 \%$ of local vegetables that have been cultivated at 16 types of local vegetables such as: Bawang Merah, Bawang leaves Cabbage, Cauliflower, chinese cabbage / mustard, Long Bean, Large Chilli, chilli, Mushroom, Tomato, eggplant, beans, cucumber, pumpkin Siam, Kale and Spinach,
\end{abstract}

Keywords: fruits and vegetables locally; Jember; food security

\section{Introduction}

Local fruits and vegetables are part of Indonesia's biodiversity, known as Mega Biodiversity Country. Indonesia including in eight mega biodiversity countries in the world, both flora and fauna spread is very wide. Biological riches include a variety of plants, animals and microbes. There are approximately 28,000 species of plants, 350,000 species of animals and 10,000 microbes that live naturally in Indonesia (Surtikanti, 2009: 124).

Indonesian forests have great potential in contributing to the provision of food for the community. From the forest there are 77 types of food sources of carbohydrates, 26 kinds of 
beans, 389 types of grains and fruits, 288 kinds of vegetables, 110 kinds of herbs and spices, 75 types of oils and fats, 40 kinds of beverage ingredients and 1260 species of medicinal plants (Kuswiyati et al in Suhardi et al, 2002). This proves that Indonesia is rich (Samantha, 2014).

Indonesia is an agricultural country that is rich in agricultural products, including fruits and vegetables locally. Indonesia even is one of the largest fruit producer in the world. But the Indonesia itself is still rarely eat fruit. FAO recommendations, ideally a fruit consumed as much as $73 \mathrm{~kg} /$ capita / year. While the new Indonesian society consumes 34.55 $\mathrm{kg} /$ capita / year. UN health agency (WHO) recommends 400 grams of fruit and vegetables per day. The American Heart Association recommends 8 servings or 4.5 cups of various types of fruits and vegetables per day. The Health Act 36 of 2009 recommended to consume 3-5 servings of vegetables and 2-3 servings of fruit per day (Saleh, 2017). Indonesian society still considers fruits and vegetables is not the main menu, but only as a food supplement only.

Diversity of local fruits and vegetables is a wealth of biodiversity is very important in life, because fruits and vegetables are one human consumption. Local fruits and vegetables contribute to food security in the region through optimizing the utilization of resources of local fruits and vegetables as a provider of food. Associated with growing food needs, the necessary efforts to increase the utilization of crop diversity to meet human needs (Pugalenthi et al, 2005). Local fruits and vegetables are a natural supermarkets for residents in the area. Local fruits and vegetables contain a variety of phytonutrient that also provide important nutrients in the area, which may not be available in the absence of local fruits and vegetables. Fruits and vegetables in general is one commodity that is necessary for humans to live a healthy life. Fruits and vegetables are sources of water and nutrients, a source of vitamins and is one of the largest natural source of antioxidants in the world (Saleh, 2017).

The nutritional content as diverse as vitamin A, B, C, potassium, iron, protein and antioxidant compounds, indicating that the cultivation and consumption of vegetables may help in dealing with malnutrition in Indonesia (Becker, 2003; Madalla et al, 2013). Local fruits and vegetables are fruits and vegetables native to the area which has been widely cultivated and consumed or fruits and vegetables that have grown long introduction and known to the public in a particular area (Suryadi and Kusumana, 2004).

Jember is an agricultural area, Jember general economy based on agriculture, the maintenance and management of natural resources to be paramount and important, so it is expected that implemented economic development oriented to the development of environmentally sound. Jember as one of the rice granary of the East Java province, showed that the agricultural sector is a sector that has a significant role. Administratively, the district of Jember is divided into 31 districts and unexplored and recorded properly. Efforts to explore and identify potential local fruits and vegetables is very important in every area. This is due to the late decision by the local fruit and vegetable fruit and vegetable import penetration to the regions, consumption patterns are changing and land conversion.

This study aimed to obtain information about the database of local fruits and vegetables are circulating and sold in the markets and centers selling fruit and vegetables in Jember. This research is part of the first year of study on Developing Local Encyclopedia of Fruits and Vegetables Jember Multimedia Based Learning as a Source of Life Sciences in Support of Food Security. 


\section{Methodology}

Exploratory research using a survey method with a qualitative approach. The research instrument consisting of pieces of observation, interviews and documentation. Surveys conducted in April - May 2018.

\subsection{Research Areas}

a) Jember district has an area of approximately $3293.34 \mathrm{~km} 2$, with a length of approximately $170 \mathrm{Km}$ coast.

b) Jember is located at an altitude of 0-3300 meters above sea level (asl), with a height of Jember urban areas approximately 87 meters above sea level (asl). Most of the territory is at an altitude of between 100 and 500 meters above sea level is $37.75 \%$. Topographical conditions indicated by the slope or elevation, most of Jember District (36.60\%) with 0 to $2 \%$ slope.

c) Climate in Jember is a tropical climate. Figures temperatures ranging from $23^{\circ} \mathrm{C}$ $31^{\circ} \mathrm{C}$.

Location survey found in 31 districts in Jember (Jember Regency, 2018), namely: Subdistrict Kencong, Gumukmas, Puger, Wuluhan, Ambulu, Tempurejo, Silo, Mayang, Mumbulsari, Jenggawah, Ajung, Rambipuji, Balung, Umbulsari, Semboro, Jombang, Sumberbaru, Embankment, Bangsalsari, Panti, Sukorambi, Arjasa, Pakusari, Kalisat, Ledokombo , Sumberjambe, Sukowono, Jelbuk, Kaliwates, Sumbersari and Patrang (Figure $1)$, The study was conducted in the markets and centers of fruits and vegetables in each district. The respondents are the local fruit and vegetable traders. Data collected includes the name of a plant, plant origin (aquaculture), morphology colors of fruits and vegetables and vegetable parts are sold. Fruits and vegetables are locally obtained and documented by the cameras and samples were taken for identification.

Figure 1. Map of Jember District (Source: Jember District Government, 2018).

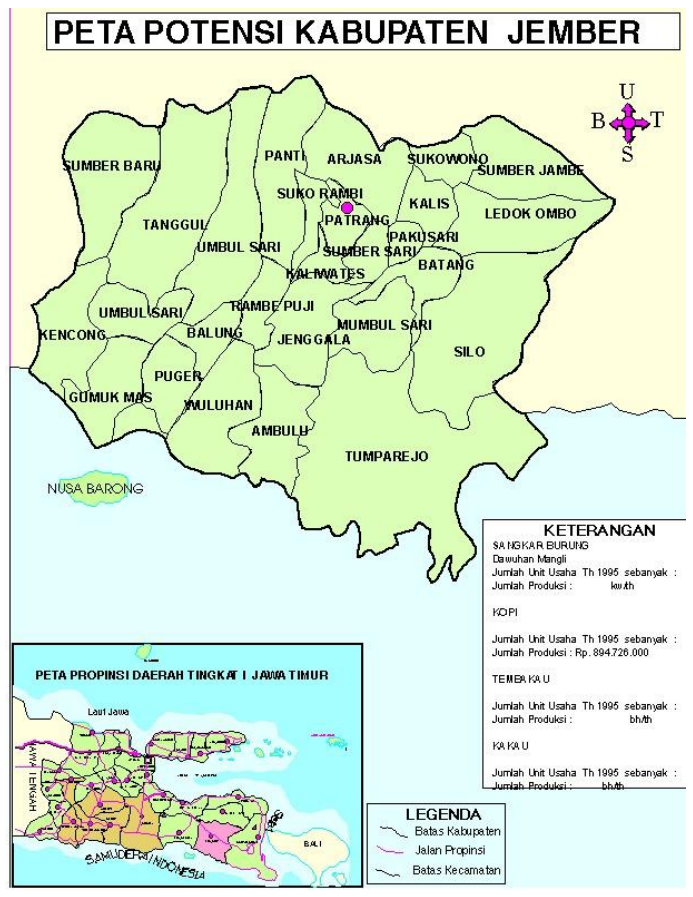




\subsection{Identification of Plant Species}

Local fruit and vegetable samples collected subsequently identified in the laboratory. Identification using book of determination: Flora of Java Volume I, II, III (Backer and Bakhuzein, 1968), A Practical Field Guide to Weeds of Rice in Asia (Caton et al, 2010), the Weed Identification (Naidu, 2012) and The Institute for Functional Medicine, 2014.

\section{Research Result}

\subsection{Fruits and Vegetables Jember Local Food Security Support}

Diversity of local fruits and vegetables Jember found in the market and the centers of the fruit are as many as 46 types of local fruits and 52 kinds of local vegetables. The survey results of local fruits and vegetables as well as distribution in 31 districts in Jember are presented in Tables 1 and 2.

Table 1. Local Fruits Found in 31 sub-districts in Jember

\begin{tabular}{|c|c|c|c|}
\hline No & Crop type & Species name & Points of Distribution (sub-district) \\
\hline 1. & Avocado. & $\begin{array}{c}\text { (Persea } \\
\text { americana }) \text {. }\end{array}$ & $\begin{array}{l}\text { Gumukmas, Puger, Wuluhan, Ambulu, } \\
\text { Silo, Mayang, Mumbulsari, Jenggawah, } \\
\text { Rambipuji, Balung, Semboro, } \\
\text { Sumberbaru, Embankment, Panti, } \\
\text { Arjasa, Pakusari, Kalisat, Ledokombo, } \\
\text { Sumberjambe, Sukowono, Jelbuk, } \\
\text { Sumbersari, and Patrang. }\end{array}$ \\
\hline 2. & Black Grape. & (Vitis vinifera). & $\begin{array}{l}\text { Ambulu, Rambipuji, Sumbersari, and } \\
\text { Patrang. }\end{array}$ \\
\hline 3. & $\begin{array}{l}\text { Belimbing } \\
\text { Manis. }\end{array}$ & $\begin{array}{l}\text { (Averrhoa } \\
\text { carambola } \mathrm{L}) \text {. }\end{array}$ & $\begin{array}{l}\text { Kencong, Gumukmas, Puger, Wuluhan, } \\
\text { Ambulu, Silo, Mayang, Mumbulsari, } \\
\text { Jenggawah, Ajung, Rambipuji, } \\
\text { Umbulsari, Semboro, Jombang, } \\
\text { Sumberbaru, Embankment, Panti, } \\
\text { Arjasa, Pakusari, Kalisat, Sumberjambe, } \\
\text { Sukowono, Jelbuk, Kaliwates, } \\
\text { Sumbersari, and Patrang. }\end{array}$ \\
\hline 4. & Starfruit. & $\begin{array}{l}\text { (Averrhoa bilimbi } \\
\text { L). }\end{array}$ & $\begin{array}{c}\text { Ambulu, Balung, embankments and } \\
\text { Kaliwates. }\end{array}$ \\
\hline 5. & Cantaloupe & (Cucumis melo). & $\begin{array}{c}\text { Ambulu, Balung, Embankment, } \\
\text { Sukowono, Kaliwates and Patrang }\end{array}$ \\
\hline 6. & $\begin{array}{l}\text { Red Dragon } \\
\text { Fruit. }\end{array}$ & $\begin{array}{l}\text { (Hylocereus } \\
\text { polyhizus). }\end{array}$ & $\begin{array}{l}\text { Ambulu, Balung, embankments and } \\
\text { Kaliwates. }\end{array}$ \\
\hline 7. & $\begin{array}{c}\text { White } \\
\text { Dragon Fruit. }\end{array}$ & $\begin{array}{l}\text { (Hylocereus } \\
\text { undatus). }\end{array}$ & $\begin{array}{c}\text { Ambulu, Embankment, Kaliwates and } \\
\text { Balung. }\end{array}$ \\
\hline 8. & Durian. & (Durio zibethinus). & $\begin{array}{l}\text { Silo, Mayang, Mumbulsari, Jenggawah, } \\
\text { Rambipuji, Balung, Semboro, }\end{array}$ \\
\hline
\end{tabular}




\begin{tabular}{|c|c|c|c|}
\hline & & & $\begin{array}{c}\text { Sumberbaru, Embankment, Bangsalsari, } \\
\text { Panti, Arjasa, Pakusari, Kalisat, } \\
\text { Ledokombo, Sumberjambe, Sukowono, } \\
\text { Jelbuk, Kaliwates, Sumbersari, and } \\
\text { Patrang. }\end{array}$ \\
\hline 9. & Guava. & $\begin{array}{c}\text { (Psidium guajava } \\
\text { L). }\end{array}$ & $\begin{array}{c}\text { Kencong, Gumukmas, Puger, Wuluhan, } \\
\text { Ambulu, Silo, Mayang, Mumbulsari, } \\
\text { Jenggawah, Rambipuji, Umbulsari, } \\
\text { Semboro, Jombang, Sumberbaru, } \\
\text { Embankment, Panti, Sukorambi, Arjasa, } \\
\text { Pakusari, Ledokombo, Sumberjambe, } \\
\text { Jelbuk, Kaliwates, Sumbersari, and } \\
\text { Patrang, }\end{array}$ \\
\hline 10. & $\begin{array}{c}\text { Guava } \\
\text { Crystals. }\end{array}$ & $\begin{array}{l}\text { (Psidium guajava } \\
\text { L). }\end{array}$ & $\begin{array}{c}\text { Ambulu, Embankment, Kaliwates and } \\
\text { Balung. }\end{array}$ \\
\hline 11. & Lime. & $\begin{array}{c}\text { (Citrus } \\
\text { aurantifolia). }\end{array}$ & $\begin{array}{c}\text { Ambulu, Embankment, Kaliwates and } \\
\text { Balung. }\end{array}$ \\
\hline 12. & $\begin{array}{l}\text { Semboro } \\
\text { orange. }\end{array}$ & (Citrus sp). & $\begin{array}{l}\text { Kencong, Gumukmas, Wuluhan, } \\
\text { Ambulu and Mayang, }\end{array}$ \\
\hline 13. & Kedondong. & (Spondias dulcis). & $\begin{array}{l}\text { Ambulu, Embankment, Kaliwates and } \\
\text { Balung. }\end{array}$ \\
\hline 14. & Lengkeng. & $\begin{array}{l}\text { (Dimocarpus } \\
\text { longanum). }\end{array}$ & Patrang \\
\hline 15. & $\begin{array}{l}\text { Manalagi } \\
\text { mango. }\end{array}$ & (Mangifera indica). & $\begin{array}{l}\text { Puger, Ambulu, Mayang, Rambipuji, } \\
\text { Sukorambi, Pakusari, and Patrang. }\end{array}$ \\
\hline 16. & $\begin{array}{l}\text { Mango } \\
\text { Gadung. }\end{array}$ & (Mangifera indica). & $\begin{array}{l}\text { Wuluhan, Silo, Mayang, Jenggawah, } \\
\text { Ajung, Balung, Semboro, Embankment, } \\
\text { Bangsalsari, Panti, Arjasa, Pakusari, } \\
\text { Ledokombo, Sumberjambe, Jelbuk, and } \\
\text { Patrang. }\end{array}$ \\
\hline 17. & Melinjo & (Gnetum gnemon). & $\begin{array}{c}\text { Puger, Ambulu, Silo, Ajung, Panti, } \\
\text { Arjasa, Pakusari, Kalisat, and } \\
\text { Sumberjambe. }\end{array}$ \\
\hline 18. & Pineapple. & (Ananas comosus). & Puger and Rambipuji \\
\hline 19. & Nangka. & $\begin{array}{c}\text { (Artocarpus } \\
\text { heterophyllus). }\end{array}$ & $\begin{array}{l}\text { Gumukmas, Puger, Wuluhan, Ambulu, } \\
\text { Silo, Mayang, Mumbulsari, Ajung, } \\
\text { Balung, Sumberbaru, Arjasa, Pakusari, } \\
\text { Kalisat, Ledokombo, Sumberjambe, } \\
\text { Jelbuk, Kaliwates, Sumbersari, and } \\
\text { Patrang. }\end{array}$ \\
\hline 20. & $\begin{array}{l}\text { Papaya } \\
\text { Thailand. }\end{array}$ & (Carica papaya L). & $\begin{array}{l}\text { Gumukmas, Puger, Wuluhan, Ambulu, } \\
\text { Tempurejo, Silo, Mayang, Mumbulsari, } \\
\text { Jenggawah, Ajung, Rambipuji, Balung, } \\
\text { Semboro, Sumberbaru, Embankment, } \\
\text { Bangsalsari, Panti, Sukorambi, Arjasa, }\end{array}$ \\
\hline
\end{tabular}




\begin{tabular}{|c|c|c|c|}
\hline & & & $\begin{array}{c}\text { Pakusari, Kalisat, Ledokombo, } \\
\text { Sumberjambe, Sukowono, Jelbuk, } \\
\text { Sumbersari, and Patrang. }\end{array}$ \\
\hline 21. & $\begin{array}{c}\text { Papaya } \\
\text { California. }\end{array}$ & (Carica papaya L). & $\begin{array}{c}\text { Gumukmas, Puger, Wuluhan, Ambulu, } \\
\text { Tempurejo, Silo, Mayang, Mumbulsari, } \\
\text { Jenggawah, Ajung, Rambipuji, Balung, } \\
\text { Semboro, Sumberbaru, Embankment, } \\
\text { Bangsalsari, Panti, Sukorambi, Arjasa, } \\
\text { Pakusari, Kalisat, Ledokombo, } \\
\text { Sumberjambe, Sukowono, Jelbuk, } \\
\text { Sumbersari, and Patrang. }\end{array}$ \\
\hline 22. & $\begin{array}{l}\text { Ambon } \\
\text { banana. }\end{array}$ & $\begin{array}{c}\text { (Musa } \\
\text { Paradisiaca). }\end{array}$ & $\begin{array}{l}\text { Kencong, Gumukmas, Puger, Wuluhan, } \\
\text { Ambulu, Tempurejo, Silo, Mayang, } \\
\text { Mumbulsari, Jenggawah, Ajung, } \\
\text { Rambipuji, Balung, Umbulsari, } \\
\text { Semboro, Jombang, Sumberbaru, } \\
\text { Embankment, Bangsalsari, Panti, } \\
\text { Sukorambi, Arjasa, Pakusari, Kalisat, } \\
\text { Ledokombo, Sumberjambe, Sukowono, } \\
\text { Jelbuk, Kaliwates, Sumbersari, and } \\
\text { Patrang. }\end{array}$ \\
\hline 23. & $\begin{array}{l}\text { Bananas } \\
\text { Barlin. }\end{array}$ & $\begin{array}{c}\text { (Musa } \\
\text { Paradisiaca). }\end{array}$ & $\begin{array}{l}\text { Kencong, Gumukmas, Puger, Wuluhan, } \\
\text { Ambulu, Tempurejo, Silo, Mayang, } \\
\text { Mumbulsari, Jenggawah, Ajung, } \\
\text { Rambipuji, Balung, Umbulsari, } \\
\text { Semboro, Jombang, Sumberbaru, } \\
\text { Embankment, Bangsalsari, Panti, } \\
\text { Sukorambi, Arjasa, Pakusari, Kalisat, } \\
\text { Ledokombo, Sumberjambe, Sukowono, } \\
\text { Jelbuk, Kaliwates, Sumbersari, and } \\
\text { Patrang. }\end{array}$ \\
\hline 24. & $\begin{array}{c}\text { Bananas } \\
\text { Kepok. }\end{array}$ & (Musa acuminata). & $\begin{array}{l}\text { Kencong, Gumukmas, Puger, Wuluhan, } \\
\text { Ambulu, Tempurejo, Silo, Mayang, } \\
\text { Mumbulsari, Jenggawah, Ajung, } \\
\text { Rambipuji, Balung, Umbulsari, } \\
\text { Semboro, Jombang, Sumberbaru, } \\
\text { Embankment, Bangsalsari, Panti, } \\
\text { Sukorambi, Arjasa, Pakusari, Kalisat, } \\
\text { Ledokombo, Sumberjambe, Sukowono, } \\
\text { Jelbuk, Kaliwates, Sumbersari, and } \\
\text { Patrang. }\end{array}$ \\
\hline 25. & $\begin{array}{l}\text { Bananas } \\
\text { Nangka. }\end{array}$ & (Musa sp L). & $\begin{array}{l}\text { Kencong, Gumukmas, Puger, Wuluhan, } \\
\text { Ambulu, Tempurejo, Silo, Mayang, } \\
\text { Mumbulsari, Jenggawah, Ajung, } \\
\text { Rambipuji, Balung, Umbulsari, }\end{array}$ \\
\hline
\end{tabular}




\begin{tabular}{|c|c|c|c|}
\hline & & & $\begin{array}{c}\text { Semboro, Jombang, Sumberbaru, } \\
\text { Embankment, Bangsalsari, Panti, } \\
\text { Sukorambi, Arjasa, Pakusari, Kalisat, } \\
\text { Ledokombo, Sumberjambe, Sukowono, } \\
\text { Jelbuk, Kaliwates, Sumbersari, and } \\
\text { Patrang. }\end{array}$ \\
\hline 26. & $\begin{array}{l}\text { Bananas } \\
\text { Raja. }\end{array}$ & (Musa sapientum). & $\begin{array}{l}\text { Kencong, Gumukmas, Puger, Wuluhan, } \\
\text { Ambulu, Tempurejo, Silo, Mayang, } \\
\text { Mumbulsari, Jenggawah, Ajung, } \\
\text { Rambipuji, Balung, Umbulsari, } \\
\text { Semboro, Jombang, Sumberbaru, } \\
\text { Embankment, Bangsalsari, Panti, } \\
\text { Sukorambi, Arjasa, Pakusari, Kalisat, } \\
\text { Ledokombo, Sumberjambe, Sukowono, } \\
\text { Jelbuk, Kaliwates, Sumbersari, and } \\
\text { Patrang. } \\
\end{array}$ \\
\hline 27. & Salak Java. & (Salacca edulis). & $\begin{array}{l}\text { Kencong, Gumukmas, Puger, Wuluhan, } \\
\text { Ambulu, Tempurejo, Silo, Mayang, } \\
\text { Mumbulsari, Jenggawah, Ajung, } \\
\text { Rambipuji, Umbulsari, Semboro, } \\
\text { Sumberbaru, Pakusari, Kalisat, } \\
\text { Sumberjambe, Jelbuk, and Sumbersari. }\end{array}$ \\
\hline 28. & Sawo. & (Manilkara zapota). & $\begin{array}{l}\text { Gumukmas, Puger, Wuluhan, Ambulu, } \\
\text { Silo, Mayang, Jenggawah, Ajung, } \\
\text { Balung, Sumberbaru, Embankment, } \\
\text { Bangsalsari, Arjasa, Pakusari, } \\
\text { Kaliwates, Sumbersari, and Patrang. }\end{array}$ \\
\hline 29. & $\begin{array}{l}\text { Watermelon } \\
\text { Red. }\end{array}$ & (Citrullus lanatus). & $\begin{array}{c}\text { Kencong, Gumukmas, Puger, Wuluhan, } \\
\text { Ambulu, Silo, Ajung, Rambipuji, } \\
\text { Balung, Umbulsari, Jombang, } \\
\text { Embankment, Bangsalsari, Panti, Arjasa, } \\
\text { Jelbuk, Kaliwates, Sumbersari and } \\
\text { Patrang. }\end{array}$ \\
\hline 30. & $\begin{array}{c}\text { Yellow } \\
\text { Watermelon. }\end{array}$ & (Citrullus lanatus). & $\begin{array}{c}\text { Kencong, Ambulu, Rambipuji, Balung, } \\
\text { Embankment, Arjasa, Kaliwates, } \\
\text { Sumbersari and Patrang }\end{array}$ \\
\hline 31. & Sirsak. & (Annona muricata). & $\begin{array}{c}\text { Gumukmas, Puger, Ambulu, Silo, } \\
\text { Mayang, Mumbulsari, Jenggawah, } \\
\text { Ajung, Rambipuji, Semboro, } \\
\text { Sumberbaru, }\end{array}$ \\
\hline 32. & Srikaya. & $\begin{array}{c}\text { (Annona aquamosa } \\
\text { L). }\end{array}$ & $\begin{array}{c}\text { Ambulu, Balung, Kaliwates, } \\
\text { Embankment, Patrang, Sumbersari, } \\
\text { Arjasa and Jelbuk. }\end{array}$ \\
\hline 33. & Sukun. & $\begin{array}{l}\text { (Artocarpus } \\
\text { communis) }\end{array}$ & $\begin{array}{l}\text { Gumukmas, Ambulu, Silo, Mayang, } \\
\text { Mumbulsari, Jenggawah, Ajung, }\end{array}$ \\
\hline
\end{tabular}




\begin{tabular}{|c|c|c|c|}
\hline & & & $\begin{array}{c}\text { Balung, Semboro, Panti, Pakusari, } \\
\text { Ledokombo, Sumberjambe, Kaliwates, } \\
\text { and Patrang. }\end{array}$ \\
\hline 34. & Large orange & (Vitis vinifera). & $\begin{array}{c}\text { Ambulu, Silo, Mayang, Jenggawah, } \\
\text { Ajung, Semboro, Pakusari, and } \\
\text { Sumbersari. }\end{array}$ \\
\hline 35. & Buni fruit. & (Antidesma bunius). & Sumbersari and Sukorambil. \\
\hline 36. & fruit Butter & $\begin{array}{l}\text { (Nerium oleander } \\
\text { L) }\end{array}$ & $\begin{array}{l}\text { Ambulu, Kencong, Wuluhan and } \\
\text { Bangsalsari. }\end{array}$ \\
\hline 37. & Ciplukan. & $\begin{array}{l}\text { (Physalis angulata } \\
\text { L). }\end{array}$ & Ambulu, Wuluhan and Sukowono. \\
\hline 38. & Duku. & $\begin{array}{c}\text { (Lansium } \\
\text { domesticum). }\end{array}$ & $\begin{array}{c}\text { Wuluhan, Silo, Mumbulsari, Jenggawah, } \\
\text { Ajung, Rambipuji, Balung, Semboro, } \\
\text { Sumberbaru, Embankment, Bangsalsari, } \\
\text { Panti, Sukorambi, Arjasa, Kalisat, } \\
\text { Ledokombo, Sumberjambe, Sukowono, } \\
\text { Jelbuk, Kaliwates, Sumbersari, and } \\
\text { Patrang. }\end{array}$ \\
\hline 39. & Rambutan. & $\begin{array}{c}\text { (Nephelium } \\
\text { lappaceum L). }\end{array}$ & $\begin{array}{l}\text { Kencong, Puger, Wuluhan, Ambulu, } \\
\text { Tempurejo, Silo, Mumbulsari, } \\
\text { Jenggawah, Ajung, Rambipuji, } \\
\text { Umbulsari, Jombang, Sumberbaru, } \\
\text { Embankment, Panti, Sukorambi, Arjasa, } \\
\text { Kalisat, Ledokombo, Sumberjambe, } \\
\text { Jelbuk, Kaliwates, and Patrang. }\end{array}$ \\
\hline 40. & Passion fruit. & (Passiflora edulis). & Mayang, Jenggawah, and Sumbersari. \\
\hline 41. & Pomegranate & $\begin{array}{c}\text { (Punica granatum } \\
\text { L). }\end{array}$ & $\begin{array}{c}\text { Kencong, Ambulu, Kaliwates, } \\
\text { Jenggawah, Ajung, Pakusari and } \\
\text { Sumbersari. }\end{array}$ \\
\hline 42. & Water apple. & (Eugenia aquea). & $\begin{array}{c}\text { Gumukmas, Puger, Ambulu, Mayang, } \\
\text { Mumbulsari, Jenggawah, Ajung, } \\
\text { Sumberbaru, Embankment, Kaliwates, } \\
\text { and Patrang. }\end{array}$ \\
\hline 43. & Mangosteen. & $\begin{array}{c}\text { (Garcinia } \\
\text { mangostana } \mathrm{L}) .\end{array}$ & $\begin{array}{l}\text { Wuluhan, Silo, Mayang, Jenggawah, } \\
\text { Ajung, Balung, Semboro, Embankment, } \\
\text { Bangsalsari, Panti, Arjasa, Pakusari, } \\
\text { Ledokombo, Sumberjambe, Jelbuk, and } \\
\text { Patrang. }\end{array}$ \\
\hline 44. & Kenitu. & $\begin{array}{l}\text { (Chrysophyllum } \\
\text { cainito). }\end{array}$ & $\begin{array}{l}\text { Ajung, Gumukmas, Rambipuji, } \\
\text { Jombang, Arjasa and Sumbersari }\end{array}$ \\
\hline 45. & Matoa. & (Pometia pinnata). & Rambipuji, Sukorambi and Sumbersari. \\
\hline \multirow[t]{2}{*}{46.} & Melon Apple & (Cucumis melo). & $\begin{array}{c}\text { Kencong, Ambulu, Rambipuji, Balung, } \\
\text { Embankment, Arjasa, Kaliwates, } \\
\text { Sumbersari and Patrang }\end{array}$ \\
\hline & Total & 46 & \\
\hline
\end{tabular}


Of the 46 types of local fruits were found, $52.17 \%$ were found to have been cultivated, namely Avocado, Grapes, Belimbing, Duku, Durian, Guava, Guava Water, Orange Semboro, Orange Large, longan, mango, mangosteen, Melinjo, Nangka, pineapple, papaya, banana, rambutan, Salak, Sawo, passionfruit, soursop and breadfruit.

Table 2. Local Vegetables Found in 31 sub-districts in Jember

\begin{tabular}{|c|c|c|}
\hline No. & Vegetables & Distribution Points \\
\hline 1. & Bean (Phaseolus vulgaris Linn) & $\begin{array}{l}\text { Kencong, Ambulu, Balung, Umbulsari, } \\
\text { Sukorambi. }\end{array}$ \\
\hline 2. & Large chilli (Capsicum annuum L.) & $\begin{array}{c}\text { Kencong, Gumuk Mas, Puger, Wuluhan, } \\
\text { Ambulu, Tempurejo, Silo, Mayang Sari } \\
\text { Mumbul, Jenggawah, Ajung, Rambipuji, } \\
\text { Bannerman Sari, Semboro, Jombang, } \\
\text { Sumberbaru, Ward Sari, Sukorambi, } \\
\text { Ledokombo, Sumber Jambe, Sukowono, } \\
\text { Patrang. }\end{array}$ \\
\hline 3. & $\begin{array}{l}\text { Cayenne pepper (Capsicum } \\
\text { frustescenns L) }\end{array}$ & $\begin{array}{c}\text { Kencong, Gumuk Mas, Puger, Wuluhan, } \\
\text { Ambulu, Tempurejo, Silo, Mayang, Mumbul } \\
\text { Sari, Jenggawah, Ajung, Rambipuji, Balung, } \\
\text { Bannerman Sari, Semboro, Jombang, } \\
\text { Sumberbaru, Embankment, Panti, Sukorambi, } \\
\text { Arjasa, Pakusari, Kalisat, Ledokombo, } \\
\text { Sumber Jambe, Sukowono, Jelbuk, } \\
\text { Sumbersari, Patrang. }\end{array}$ \\
\hline 4. & Pigeonpea (Cajanus cajan L.) & Kalisat, Jenggawah and embankments. \\
\hline 5. & $\begin{array}{c}\text { Long beans (Vigna unguiculata } \\
\text { sesquipedali) }\end{array}$ & $\begin{array}{l}\text { Kencong, Gumuk Mas, Puger, Wuluhan, } \\
\text { Ambulu, Tempurejo, Silo, Mayang, } \\
\text { Mumbulsari, Jenggawah, Ajung, Rambipuji, } \\
\text { Balung, Umbulsari, Semboro, Jombang, } \\
\text { Sumberbaru, Embankment, Bangsalsari. }\end{array}$ \\
\hline 6. & $\begin{array}{l}\text { Cowpeas (Psophocarpus } \\
\text { tetragonolobus L.) }\end{array}$ & $\begin{array}{l}\text { Kaliwates, Kalisat, Jenggawah, Embankment, } \\
\text { Ambulu, Mumbulsari, Sumbersari and Panti. }\end{array}$ \\
\hline 7. & Soy (Glycine max) & Embankment, Kalisat and Kaliwates. \\
\hline 8. & Kluwih (Artocarpus camansi) & $\begin{array}{c}\text { Kaliwates, Kalisat, Jenggawah and } \\
\text { embankments. }\end{array}$ \\
\hline 9. & Kondur(Benincasa hispida) & Kaliwates \\
\hline 10. & Keratok (Phaseolus lunatus) & $\begin{array}{l}\text { Kalisat. } \\
\end{array}$ \\
\hline 11. & $\begin{array}{l}\text { pumpkin China (Lagenaria } \\
\text { siceraria) }\end{array}$ & $\begin{array}{l}\text { Kaliwates, Kalisat, Jenggawah and } \\
\text { embankments. }\end{array}$ \\
\hline 12. & $\begin{array}{l}\text { Summer squash (Cucurbita } \\
\text { moschata Duschesne) }\end{array}$ & $\begin{array}{c}\text { Kaliwates, Kalisat, Jenggawah and } \\
\text { embankments. }\end{array}$ \\
\hline 13. & Chayote (Sechium edule) & Ledokombo. \\
\hline 14. & cucumber (Cucumis sativus L.) & $\begin{array}{c}\text { Gumuk Mas, Puger, Ambulu, Mayang, } \\
\text { Mumbulsari, Jenggawah, Ajung, Rambipuji, }\end{array}$ \\
\hline
\end{tabular}




\begin{tabular}{|c|c|c|}
\hline & & $\begin{array}{c}\text { Balung, Umbulsari, New Source, Sukorambi, } \\
\text { Ledokombo, Sumbersari }\end{array}$ \\
\hline 15. & okra (Abelmoschus esculentus L.) & Kaliwates \\
\hline 16. & Oyong / gambas (Luffa acutangula) & $\begin{array}{l}\text { Jenggawah, Embankment, Kalisat and } \\
\text { Kaliwates. }\end{array}$ \\
\hline 17. & Pare (Momordica charantia) & $\begin{array}{l}\text { Puger, Wuluhan, Silo, Ajung, Jenggawah, } \\
\text { Embankment, Kalisat and Kaliwates. }\end{array}$ \\
\hline 18. & petai china (Laucaen gluca) & Kaliwates and Kalisat. \\
\hline 19. & Pete (Parkia speciosa) & $\begin{array}{c}\text { Ambulu, Tempurejo, Silo, Mayang, } \\
\text { Rambipuji, Arjasa, Pakusari, Kalisat, } \\
\text { Ledokombo, Sumberjambe, Sukowono and } \\
\text { Kaliwates. }\end{array}$ \\
\hline 20. & Tekokak (Torvum Solanum sp) & Kaliwates, Kalisat and embankments. \\
\hline 21. & Eggplant (Solanum melongena) & $\begin{array}{l}\text { Kencong, Gumuk Mas, Ambulu, Tempurejo, } \\
\text { Mayang, Mumbulsari, Jenggawah, Ajung, } \\
\text { Rambipuji, Balung, Umbulsari, Jombang, } \\
\text { New Source, Embankment, Ward Sari, Sari } \\
\text { Nail, Kalisat, Ledokombo, Sumberjambe, } \\
\text { Jelbuk, Sumbersari. }\end{array}$ \\
\hline 22. & $\begin{array}{l}\text { eggplant Wren (Solanum } \\
\text { melongena) }\end{array}$ & $\begin{array}{l}\text { Kencong, Gumuk Mas, Ambulu, Tempurejo, } \\
\text { Mayang, Mumbulsari, Jenggawah, Ajung, } \\
\text { Rambipuji, Balung, Umbulsari, Jombang, } \\
\text { New Source, Embankment, Ward Sari, Sari } \\
\text { Nail, Kalisat, Ledokombo, Sumberjambe, } \\
\text { Jelbuk, Sumbersari. }\end{array}$ \\
\hline 23. & Tomato (Solanum lycopersicum L.) & $\begin{array}{c}\text { Gumus Mas, Ambulu, Silo, Mayang, } \\
\text { Jenggawah, Ajung, Rambipuji, New Source, } \\
\text { Sukorambi, Kalisat, Ledokombo, Sumber } \\
\text { Jambe }\end{array}$ \\
\hline 24. & $\begin{array}{l}\text { Green spinach (Amaranthus } \\
\text { spinosus) }\end{array}$ & Panti, Sukorambi, Ledokombo, Kaliwates \\
\hline 25. & $\begin{array}{l}\text { Red spinach (Amaranthus } \\
\text { gangeticus L.) }\end{array}$ & Panti, Sukorambi, Ledokombo, Kaliwates \\
\hline 26. & $\begin{array}{l}\text { Onion leaves (Cleome } \\
\text { rutidospermae) }\end{array}$ & Wuluhan, Jenggawah, Jombang. \\
\hline 27. & Boboan (Allium fistulosum L) & Jenggawah. \\
\hline 28. & $\begin{array}{l}\text { Caisin / mustard noodles (Brassica } \\
\text { campestris L-spp.) }\end{array}$ & $\begin{array}{c}\text { Gumuk Mas, Balung, Embankment, } \\
\text { Sukorambi, Kaliwates. }\end{array}$ \\
\hline 29. & $\begin{array}{c}\text { Leaves Katu (Sauropus } \\
\text { androgynus) }\end{array}$ & Jenggawah, embankments and Kaliwates. \\
\hline 30. & $\begin{array}{c}\text { Moringa leaves(Moringa oleifera } \\
\text { Lam.) }\end{array}$ & Kaliwates and Bangsalsari. \\
\hline 31. & Bay leaf (Syzygium polyanthum) & Kaliwates, Kalisat and embankments. \\
\hline 32. & Cassava leaves (Manihot esculenta) & $\begin{array}{c}\text { Kaliwates, Kalisat, Jenggawah and } \\
\text { embankments. }\end{array}$ \\
\hline
\end{tabular}




\begin{tabular}{|c|c|c|}
\hline 33. & Papaya leaf (Carica papaya L.) & $\begin{array}{l}\text { Kencong, Jenggawah, Embankment, Kalisat } \\
\text { and Kaliwates. }\end{array}$ \\
\hline 34. & genjer (Limnocharis flava) & $\begin{array}{l}\text { Kencong, Jenggawah, Embankment, Kalisat } \\
\text { and Kaliwates. }\end{array}$ \\
\hline 35. & $\begin{array}{c}\text { Gunda(Sphenoclea zeylanica } \\
\text { Gaertn) }\end{array}$ & Kaliwates \\
\hline 36. & $\begin{array}{l}\text { Junggulan(Erechtitesvalerianifolia } \\
\text { Raf.) }\end{array}$ & Kaliwates \\
\hline 37. & kale water (Ipomea aquatic) & Panti, Sukorambi, Ledokombo, Kaliwates \\
\hline 38. & kale land (Ipomea reptans Poir) & Panti, Sukorambi, Ledokombo, Kaliwates \\
\hline 39. & Basil (Ocimum sanctum L.) & $\begin{array}{c}\text { Kaliwates, Kalisat, Jenggawah and } \\
\text { embankments. }\end{array}$ \\
\hline 40. & Marigolds (Cosmos caudatus) & $\begin{array}{l}\text { Kaliwates, Kalisat, Jenggawah and } \\
\text { embankments. }\end{array}$ \\
\hline 41. & Cabbage (Brassica oleracea) & $\begin{array}{c}\text { Puger, Wuluhan, Ambulu, Jenggawah, } \\
\text { Balung, Panti }\end{array}$ \\
\hline 42. & Fern (Diplazium esculentum) & $\begin{array}{c}\text { Kaliwates, Kalisat, Jenggawah and } \\
\text { embankments. }\end{array}$ \\
\hline 43. & Sawi (Brassica pekinensia L.) & $\begin{array}{l}\text { Kaliwates, Kalisat, Jenggawah and } \\
\text { embankments. }\end{array}$ \\
\hline 44. & watercress (Nasturtium spp.) & $\begin{array}{c}\text { Kaliwates, Kalisat, Jenggawah and } \\
\text { embankments. }\end{array}$ \\
\hline 45. & Celery (Apium graveolens L.) & $\begin{array}{c}\text { Kaliwates, Kalisat, Jenggawah, Patrang, } \\
\text { Jelbuk, Mumbulsari, Balung and } \\
\text { embankments. }\end{array}$ \\
\hline 46. & lettuce leaves (Lactuca sativa L.) & Kaliwates, Jenggawah and embankments. \\
\hline 47. & Cauliflower (Brassica oleracea) & Ambulu \\
\hline 48. & papaya flower(Carica papaya L.) & Kaliwates, Jenggawah and embankments. \\
\hline 49. & Flowers Turi (Sesbania grandiflora) & Kaliwates and embankments. \\
\hline 50. & $\begin{array}{c}\text { Shallots (Allium Colocasia } \\
\text { esculenta) }\end{array}$ & Wuluhan, Jenggawah, Jombang \\
\hline 51. & Taro (Colocasia esculenta) & $\begin{array}{c}\text { Kaliwates, Kalisat, Jenggawah and } \\
\text { embankments. }\end{array}$ \\
\hline 52 & mushroom & $\begin{array}{l}\text { Wuluhan, Silo, Ajung, Rambipuji, Balung, } \\
\text { Panti, Patrang. }\end{array}$ \\
\hline
\end{tabular}

Of the 52 types of local vegetables are found, $30.8 \%$ found already cultivated, Namely: Shallots (Allium ascalonicum), Onion leaves (Allium fistulosum L), Cabbage (Brassica oleracea), cauliflower (Brassica oleracea), Chinese cabbage / mustard (Brassica campestris L-spp.), Long Beans (Vigna unguiculata sesquipedali), Large Chilli (Capsicum annuum L.), chilli (Capsicum frustescenns L.), Mushroom, Tomato (Solanum lycopersicum L.), eggplant (Solanum melongena), beans (Phaseolus vulgaris Linn), Cucumber (Cucumis sativus L.), Pumpkin Siam (Sechium edule), kale (Ipomea sp.) And Spinach ((Amaranthus spinosus)). 
Research has found that 46 kinds of local fruits and 52 kinds of local vegetables. Documentation of the diversity of local fruit Jember found in more than 20 sub-districts can be seen in Figure 2.

Figure 2. Local Fruits Kab. Jember found in more than 20 districts

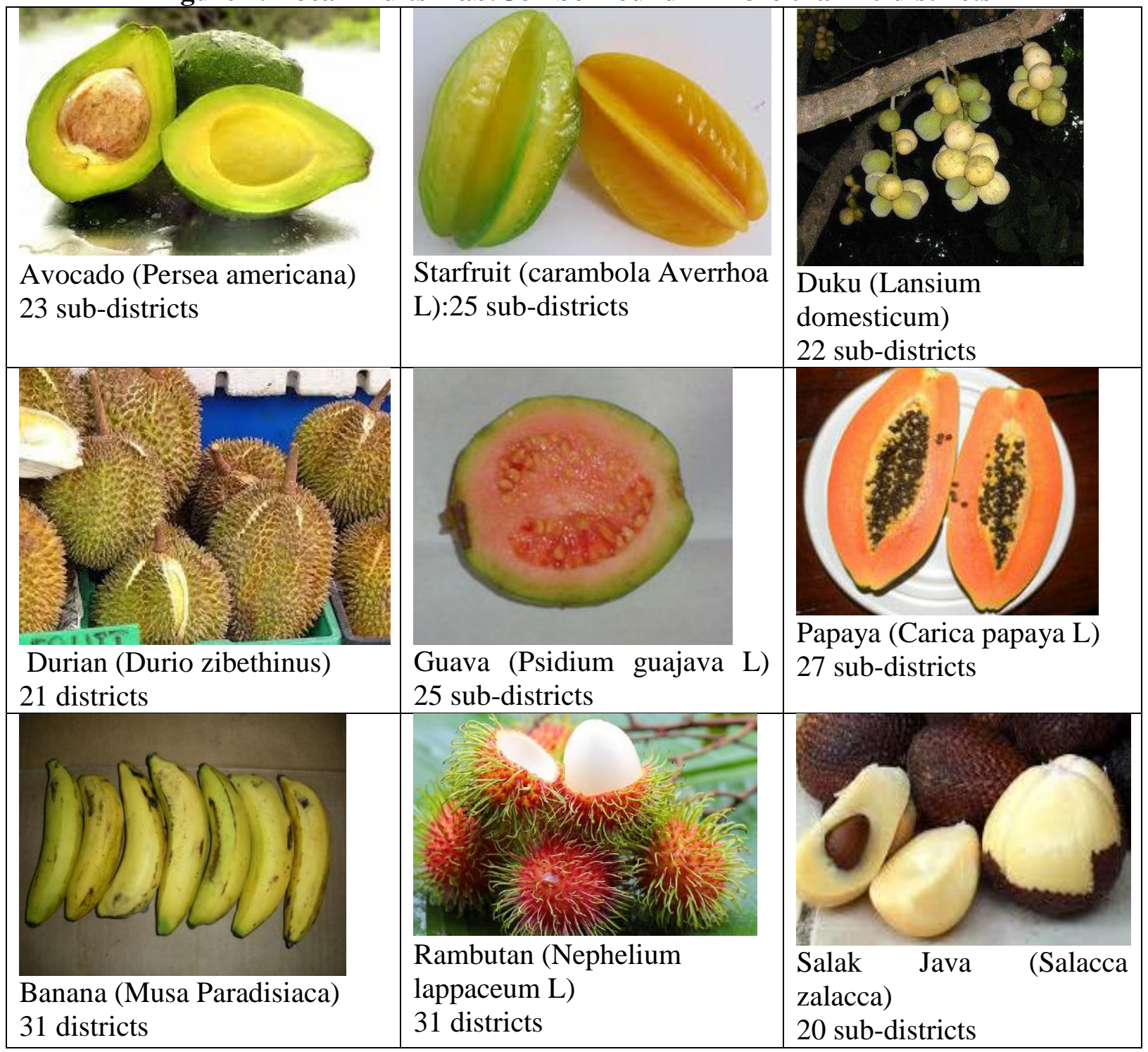

The documentation of the diversity of local vegetables Jember found in more than 12 districts can be seen in Figure 3. 
Figure 3. Local Vegetable Kab. Jember found in more than 12 districts

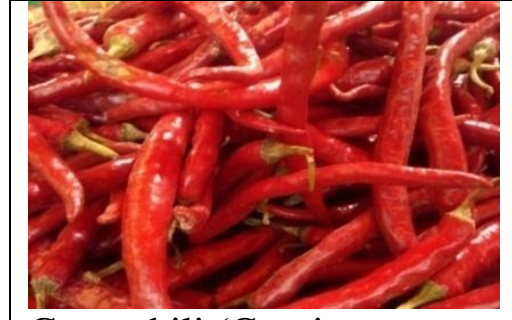

Great chili (Capsicum annuum L) 22 subdistricts

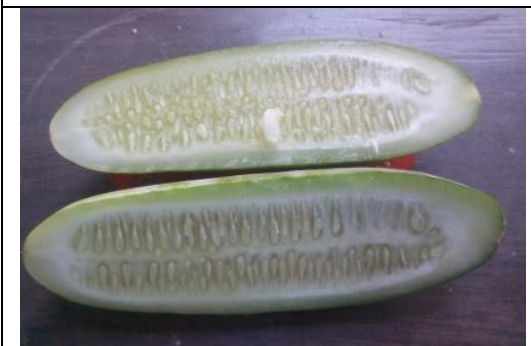

Cucumber (Cucumis sativus L.) 14 districts

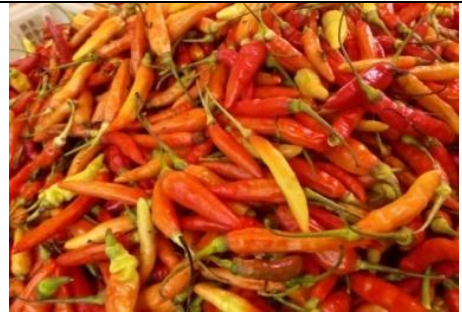

Cayenne pepper (Capsicum frustescenns L)

29 sub-districts

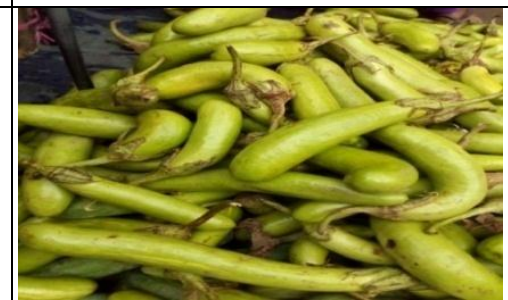

Eggplant (Solanum melongena) 22 sub-districts

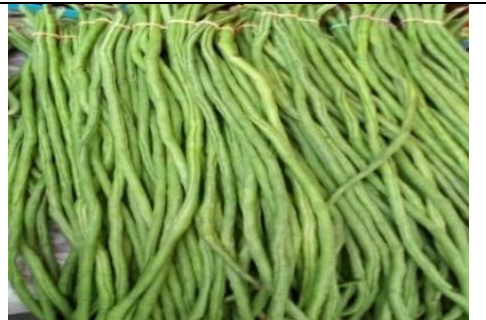

Long beans (Vigna unguiculata) 18 districts

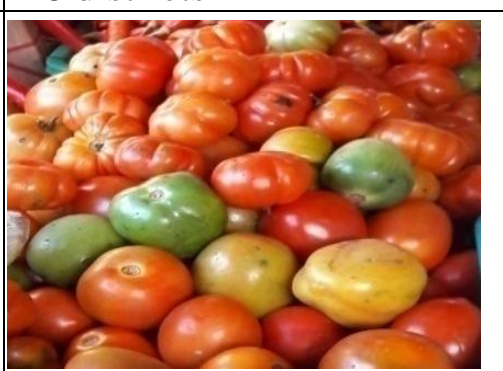

Tomato (Solanum lycopersicum L): 12 districts

The typical fruit found in Jember namely kenitu fruit, which is found in the 6 districts, the District Ajung, Gumukmas, Rambipuji, Jombang, Arjasa and Sumbersari, Can be seen in Figure 4.

Figure 4. Typical Fruit Kenitu (Chrysophyllum cainito): 6 districts

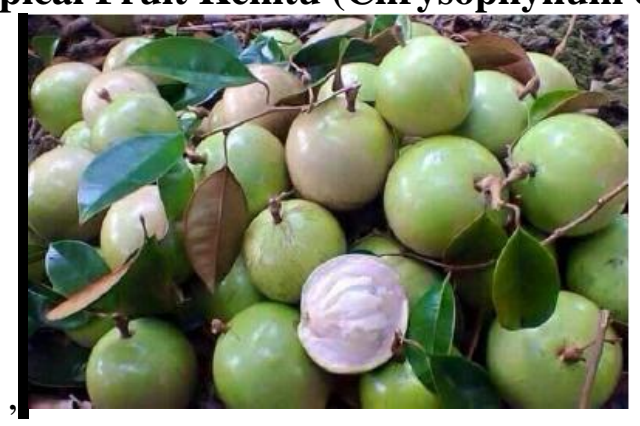

\subsection{The color spectrum Local Fruits in Jember}

Based on the research results owned fruit color spectrum in Jember has a diversity of colors, namely red, green, yellow, orange (orange), blue / purple / black and white / tan / brown. Colored fruits contain phytochemicals (phytonutrients) are different from each other. phytochemical compounds are compounds found in fruits that give it flavor, aroma, or a distinctive color on the fruit. 
Figure 5. Spectrum of Colors in Jember Local Fruits

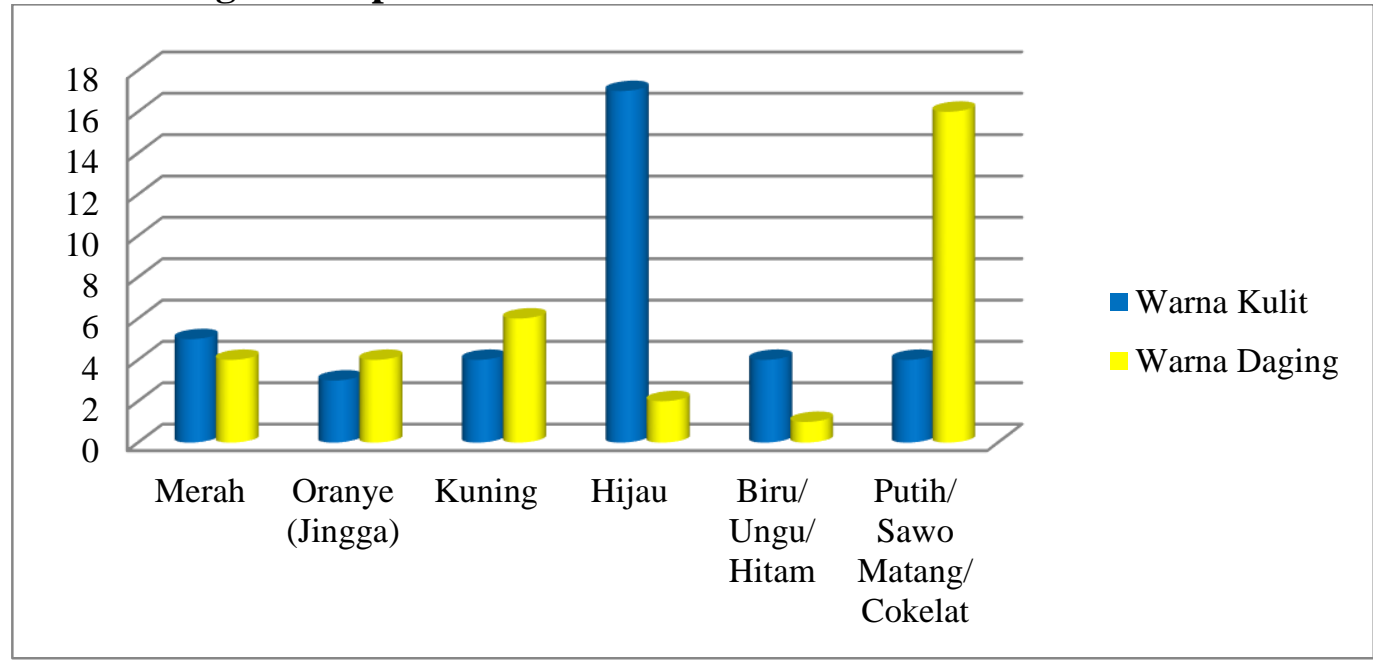

Based on the analysis of Figure 5, the local fruit found in Jember has a diversity of skin color or of different meats. The picture above shows that the green color on the skin of the fruit is the contribution of the color most acquired as many as 17 varieties of fruit, including avocado, green grapes, guava, pink crystals, lime, orange Semboro, kedondong, kenitu, cherry, mango, apple melon, watermelon red, yellow watermelons, soursop, sugar apple, breadfruit and banana, while the contribution of the orange in the fruit skin color contribution that little can be found obtained by 3 local fruit species Jember, namely pineapple, papaya, and citrus. Contributions are white / tan / brown in the flesh of the fruit is the contribution of the colors most acquired as many as 16 species of fruit, including Berry, Duku, pink crystals, kedondong, kenitu, cherry, longan, mangosteen, betel nut, rambutan, bark, fruit white dragon, breadfruit, soursop, sugar apple, and brown, while the contribution of the blue / purple / black is the color a little contribution found in Jember local fruit, dragon fruit red.

\subsection{Color Vegetable Fruit, Foliage, Flowers and Bulbs}

Figure 6. Percentage of Total Color Vegetables Fruits and Tubers by Color Skin, Flesh and Color Seeds

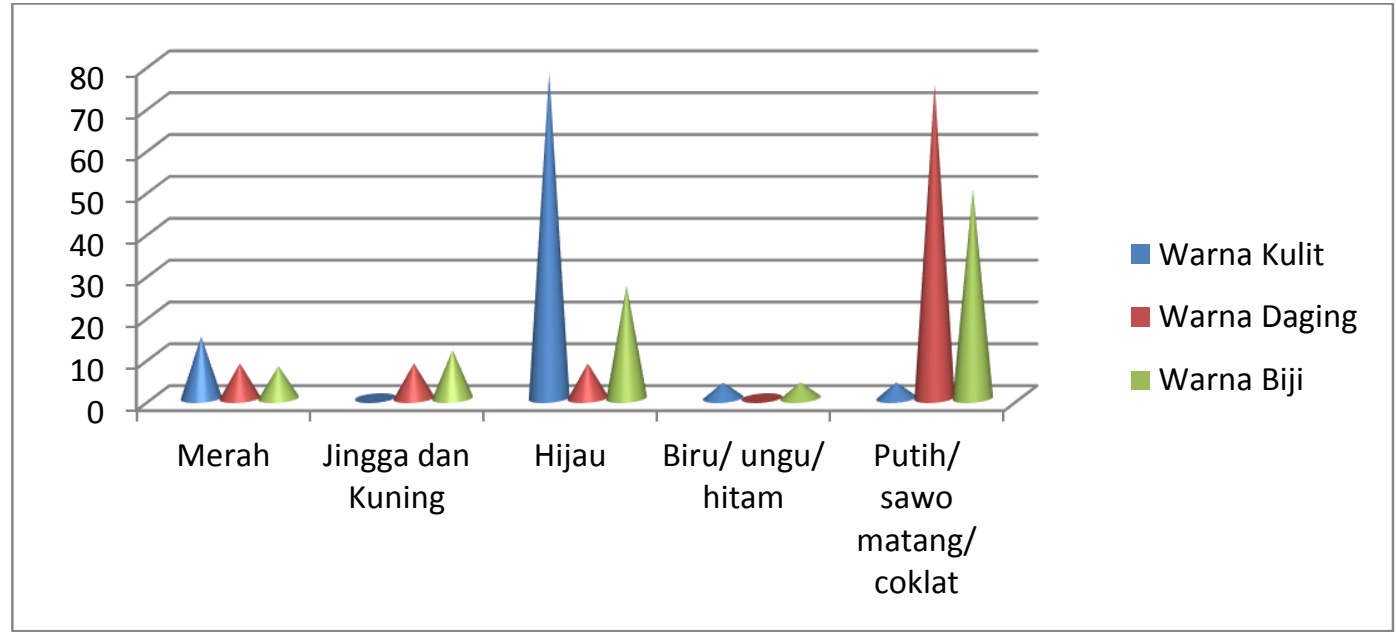


Based on the percentage of fruit and tuber vegetables based on the color of skin, flesh and seed color at most that green and white / tan / brown. Skin color with red color as much as 4 vegetables, orange and yellow as 0,21 vegetable green, purple 1 vegetable and skin colors of white and brown as much as 1 . Color red meat is divided into as many as 1 vegetable, orange and yellow 1 , Green 1 , blue / purple / black 0 , and most vegetable meat color is white as many as nine vegetables. Seed color red is divided into as many as 2 vegetables, orange and yellow 3, Green 7, the purple one, and most color is white which is about 13 vegetables.

\section{Discussion}

The results of the exploration of local fruits and vegetables sold in markets and centers of fruits and vegetables in 31 districts in Jember, found 46 kinds of local fruits and 52 kinds of local vegetables. Local fruits and vegetables have potential as a source of germplasm flora that is able to support the local food needs. Therefore, local fruits and vegetables is one of alternative food sources of qualified providers.

Local fruits and vegetables is a plant that has been known and cultivated society, but not documented / no database on the local fruit and vegetables. Though local fruit has a fresh taste with nutritional content therein. People still prefer imported fruit with different strengths. Need to cultivate a love of fruits and vegetables locally through education. Diversification based on local fruits and vegetables is an alternative that can improve nutrition and can solve the problems of malnutrition and food security. Vitamins and minerals contained in fruits and vegetables are rich in food resources that are beneficial to health, development and growth. Although the needs are relatively few, but the function of vitamins and minerals can hardly be replaced so that insufficient consumption of these substances become essential (Mohammed, 2015). Fruit is one of the agricultural commodities that contributed substantially to the diversity of food and the availability of public nutrition because it contains fiber, vitamins and minerals (Indriani, 2015). Vegetables and fruits are a source of dietary fiber that are easily found in foodstuffs. Food consumption patterns in Indonesia caused a shortage of the consumption of vegetables and fruits in almost all provinces in Indonesia. This causes a shift in the pattern of infectious diseases into degenerative diseases and metabolic (Santoso, 2011).

The world's population by $75 \%$ indicating fruit and vegetable consumption as has been recommended by WHO. WHO recommendations for the consumption of fruit and vegetables each day is 5-9 servings (400 grams). In Indonesia, the intake of fruits and vegetables only 2.5 servings (MoH RI, 2014). Around the world, 1.7 million estimated deaths per year are associated with low intake of fruits and vegetables (Miller et al, 2016).

In this study, it was found 46 types of local fruits and 52 kinds of local vegetables spread over 31 districts in Jember. There are 24 types of local fruits and 16 kinds of local vegetables have been cultivated. Found 9 types of local fruit available in more than 20 districts, namely avocado, star fruit, Duku, durian, guava, papaya, banana, rambutan and bark. There are 6 types of local vegetables that are available in more than 12 districts, namely large chilli, cayenne pepper, string beans, cucumber, eggplant and tomatoes. This discovery shows that some markets and centers of the fruit has a diversity of local fruits and vegetables are high. 
The study of 18 countries in 5 continents, illustrating that the availability of fruits and vegetables throughout the region is related to feed its population (Miller et al, 2016). The colors of the fruits and vegetables of different colors such as blue, purple, red, green, white and yellow-orange. Each color indicates the presence of certain phytochemical compounds are efficacious for preventing various diseases (Astawan, 2008). Each color contains different phytonutrients, so that optimal health can be realized if the intake of fruits and vegetables of different colors meet the recommended servings. Fruits and vegetables are rich in antioxidant phytonutrients have 64 times more than animal foods (Amagram, 2014). A world population that consumes very little fruit and vegetables, both the number and diversity, potentially lower its phytonutrient intake. Phytonutrients in fruits consist of a variety of colors, including red, green, yellow, orange (orange), blue / purple, and white / brown which serves to determine the benefits in health, communities are encouraged to consume fruits with color varies daily ( The Institute for Functional Medicine, 2014). According Astawan (2008) suggest that consuming public at least five food groups everyday color, namely red, white, blue or purple, yellow, and green. Each color indicates the presence of certain phytochemical compounds are efficacious for preventing various diseases.

Diversity fruit found in Jember will contribute to the availability fitonutrient for Jember Regency society. The availability of local fruits are stable for fruits that knows no season. Availability of local vegetables are stable for 16 kinds of vegetables that have been cultivated. What kind of fruit is a fruit typical of that found kenitu, the spreading can be found in the 6 districts, the District Ajung, Gumukmas, Rambipuji, Jombang, Arjasa and Sumbersari. Among local fruit, found 52.2,\% sold derived from the cultivation and 30.8\% coming from the local vegetable cultivation. Of the 52 types of local vegetables, which are often used in the form of fruit vegetables 23, 23 leaves, 3 flowers and 2 vegetable bulbs. This is consistent with some studies finding that the leaves are part of the vegetables are often consumed (Susanti, 2015; Chotimah et al, 2013).

Database creation of local fruits and vegetables in Jember, for the first time made through this research. Results of research have discovered 46 types of local fruits and 52 kinds of local vegetables can contribute to food security in Jember.

\section{Bibliography}

Amagram (2014). Global Phytonutrient Report by Nutrilite. Amagram magazine. January 11th. Jakarta.

Astawan, Made; Love, Andreas Leomitro. (2008). Efficacy Colorful food. Jakarta: PT Gramedia Pustaka Utama.

Becker, K., Afuang, W., Siddhuraju, P. (2003). Nutritional Comparative Evaluation of Raw, Methanol Extracs of Moringa (Moringa oleifera Lam.) Leaves on Growth Performance and Feed Utilization in Nile Tilapia (Oreochromis niloticus L.). Aquaculture Research 34, 13, 1147-1159.

Caton, BP, Mortimer, M., Hill, JE, Johnson, DE (2010). A Practical Field Guide to Weeds of Rice in Asia. Philippines: International Rice Research Institute.

Indriani, Y. (2015). Nutrition and Food. Bandar Lampung: Aura. 
Ministry of Health of the Republic of Indonesia. (2014). Balanced Nutrition Guidelines. Jakarta.

Madalla, N., Agbo, NW, Jauncey, K. (2013). Evaluation of Aqueous Extracted Moringa Leaf Meal as a Protein Source for Nile Tilapia Juveniles. Tanzania Journal of Agricultura Science, 12, 1, 53-64.

Miller, Victoria., Yusuf, Salim., Chow, Clara K., Dehghan, Mahshid., Corsi, Daniel J; Lock, Karen., Popkin, Barry., Rangarajan, Sumathy., Khatib, Rasha., Lear, Scott A., Mony, Prem., Kaur, Manmeet., Mohan, Viswanathan., Vijayakumar, Krishnapillai., Gupta, Rajeev. , Kruger, Annamarie., Tsolekile, Lungiswa., Mohammadifard, Noushin., Rahman, Omar., Rosengren, Annika., Avezum, Alvaro., Orlandini, Andrés., Ismail, Noorhassim., Lopez-Jaramillo, Patricio., Yusufali, Afzalhussein., Karsidag, Kubilay. Iqbal, Romaina., Chifamba, Jephat., Oakley, Solange Martinez., Ariffin, Farnaza., Zatonska, Katarzyna., Poirier, Paul., Wei Li, Jian Bo., Hui Chen, Xu, Liu., Xiulin, Bai., Teo, Koon., cashew nut, Andrew. (2016). Availability, aff ordability, and consumption of fruits and vegetables in 18 countries across income levels: findings from the Prospective Urban Rural Epidemiology (PURE) study. Glob Health Lancet 2016; 4: e695-703.http://dx.doi.org/10.1016/

Mohammad, Andika; Madanijah, Siti. (2015). Consumption of Fruit and Vegetable Childhood Primary School in Bogor. Journal of Nutrition 10 (1): 71-76.

Naidu, VSGR (2012). Handbook on Weed Identification. Directorate of Weed Science Research. India: Jabalpur.

Pugalenthi, M., Vadivel, V., Siddhuraju, P. (2005). Alternative Food / Feed Perspectives of an underutilized Legume Mucuna pruriens Var. Utilis - a review. Plants Foods for Human Nutrition, 60, 201-218.

Saleh, AR (2017). Against Research Map 12 Type Local Fruits Indonesia At Theses and Dissertations Graduate University. Bogor Agricultural University. DOI.10.13140 / RG.2.2.17337.36961

Samantha. (2014). Realize Food Sovereignty with Biodiversity. (On line), (http://nationalgeographic.co.id/berita/2014/08/wujudkan-kedaulatan-pangandengan-keanekaragaman-hayati, Accessed March 21, 2017).

Santoso, A. (2011). Food fiber (Dietary Fiber) and Benefits for Health. Magistra No. 75 Th. XXIII, in March.

Suhardi, SA, Sudjoko and Minaminingsih. (2002). Forests and gardens as a Source of National Food. Jakarta: Canisius.

Surtikanti, HK (2009). Environmental Biology. Bandung: Prisma Press Prodaktama.

Suryadi., Kusmana. (2004). Vegetables recognize Indijenes. Monograph No. 25. Bandung: Vegetable Crops Research Institute.

Susanti, H. (2015). Ethnobotanical Study for Swamp Local Vegetables at Martapura Market of South Borneo. Ziraa'ah, 40, 2, 140-144.

The Institute for Functional Medicine. (2014). phytonutrient Spectrumhttp://www.thehealthedgepodcast.com/wpcontent/uploads/2015/07/Phytonu trient-Spectrum-Comprehensive-Guide.pdf 